\title{
Correction to: A sharp Bernstein-type inequality and application to the Carleson embedding theorem with matrix weights
}

\section{Daniela Kraus ${ }^{1} \cdot$ Annika Moucha ${ }^{1} \cdot$ Oliver Roth $^{1}$ (DD}

Published online: 3 March 2022

(c) Springer Nature Switzerland AG 2022

\section{Correction to: Analysis and Mathematical Physics (2022) 12:40 https://doi.org/10.1007/s13324-021-00639-5}

The original published version of this article contained a number of typos that were introduced in the typesetting process by the publisher. This update corrects those errors.

Publisher's Note Springer Nature remains neutral with regard to jurisdictional claims in published maps and institutional affiliations.

The original article can be found online at https://doi.org/10.1007/s13324-021-00639-5.

Oliver Roth

roth@mathematik.uni-wuerzburg.de

Daniela Kraus

dakraus@mathematik.uni-wuerzburg.de

Annika Moucha

annika.moucha@mathematik.uni-wuerzburg.de

1 Department of Mathematics, University of Würzburg, Emil Fischer Strasse 40, 97074 Würzburg, Germany 